2 Hussain B, Saleh GM, Sivaprasad S, Hammond CJ. Changing from Snellen to LogMAR: debate or delay? Clin Exp Ophthalmol 2006; 34: 6-8.

3 Bailey I, Lovie J. New design principles for visual acuity letter charts. Am J Optom Physiol Opt 1976; 53: 740-745.

4 Laidlaw DAH, Abbott A, Rosser DA. Development of a clinically feasible logMAR alternative to the Snellen chart: performance of the compact reduced logMAR visual acuity chart in amblyopic children. Br J Ophthalmol 2003; 87: 1232-1234.

5 Talks SJ, Setty R, Clarke L. The incidence and outcome of photodynamic therapy for macular degeneration in the Northern region of the UK. Eye 2004; 18: 588-594.

6 Bressler NM. Photodynamic therapy of subfoveal choroidal neovascularization in age-related macular degeneration with verteporfin: two-year results of 2 randomized clinical trials-tap report 2. Arch Ophthalmol 2001; 119: 198-207.

E Doyle and AG Casswell

Sussex Eye Hospital, Eastern Road, Brighton BN2 5BF, UK

Correspondence: E Doyle,

Tel: + 44 (0) 7799448543;

Fax: + 44 (0) 8714338914

E-mail: EdRachie@btinternet.com

The authors have no commercial interests in any products mentioned in this article

Eye (2007) 21, 284-285. doi:10.1038/sj.eye.6702532;

published online 4 August 2006

Sir,

\section{Reply to Doyle et al}

We would like to thank Doyle et al for their interest in our reported series of four cases. ${ }^{1}$ We agree that logMAR visual acuity measurements should be encouraged especially in situations where measurements using a Snellen chart may not be sensitive enough to recognise a change in the acuity. In keeping with the standard practice we routinely record $\log$ MAR visual acuities for all patients undergoing PDT. In the text of our report we have reported the decline in visual acuity in terms of the drop in the number of letters read on the $\log$ MAR chart. This is similar to the way results were summarised in the TAP study ${ }^{2}$ where reduction of visual acuity by fewer than 15 letters over a 24-month period was considered as beneficial effect of the treatment. Furthermore, although one of our patients lost only 14 letters, this happened rapidly over a 9-month period in spite of closure of the CNV which is not strictly comparable with the beneficial outcome reported in the TAP study.

Doyle et al have said that nowhere in the paper is the actual $\log$ MAR visual acuity shown. We would like to draw their attention to Figures 2 and 3 in our report that clearly mention the actual $\log$ MAR visual acuity values for that case. We feel that using the reciprocal of actual $\log$ MAR values appropriately illustrates graphically in Figure 1 the steep decline in visual acuity as a downward slope. We do not think that adding a table showing logMAR visual acuities would have added to our message from this small case series that in certain cases visual acuity could decline fairly rapidly in spite of a 'successful' PDT treatment.

\section{References}

1 Bhatnagar A, Musadiq M, Yang YC. Late-onset visual decline following successful treatment of subfoveal choroidal neovascularisation with photodynamic therapy. Eye 2006; 20: 491-493.

2 Treatment of Age-Related Macular Degeneration with Photodynamic Therapy (TAP) Study Group. Photodynamic therapy of subfoveal choroidal neovascularisation in age related macular degeneration with verteporfin. Two-year results of two randomised clinical trials-TAP report 2. Arch Ophthalmol 2001; 119: 198-207.

\section{A Bhatnagar, M Musadiq and YC Yang}

Wolverhampton Eye Infirmary, Compton Road, Wolverhampton, WV3 9QR, UK

Correspondence: A Bhatnagar, Wolverhampton Eye Infirmary, Compton Road, Wolverhampton, WV3 9QR UK

Tel: + 447958 582871;

Fax: + 441902645018.

E-mail: bhatnagar_ajay@btopenworld.com

Eye (2007) 21, 285. doi:10.1038/sj.eye.6702534; published online 4 August 2006

Sir,

Intravitreal triamcinolone staining observation of residual undetached cortical vitreous after posterior vitreous detachment

The use of intravitreal triamcinolone to demonstrate areas of undetached vitreous ${ }^{1}$ has gained in popularity in recent years. The authors have not clarified whether their 